\title{
The Effect of GRACE Scores on Prediction of 30-day Cardiovascular Adverse Events in Patients with Acute Chest Pain
}

\author{
Zhenhua Huang $^{\dagger}$, Qianlin Gu ${ }^{\dagger}$, Hong Zhan, Zhen Yang, Yuee Chen* \\ Emergency Department, The First Affiliated Hospital of Sun Sat-sen University, Guangzhou, China \\ Email address: \\ 13600086202@139.com (Yuee Chen) \\ ${ }^{*}$ Corresponding author \\ $\dagger$ Zhenhua Huang and Qianlin Gu are co-first authors.
}

\section{To cite this article:}

Zhenhua Huang, Qianlin Gu, Hong Zhan, Zhen Yang, Yuee Chen. The Effect of GRACE Scores on Prediction of 30-day Cardiovascular Adverse Events in Patients with Acute Chest Pain. American Journal of Clinical and Experimental Medicine. Vol. 8, No. 1, 2020, pp. 1-5. doi: 10.11648/j.ajcem.20200801.11

Received: January 31, 2020; Accepted: February 18, 2020; Published: February 26, 2020

\begin{abstract}
To investigate the effect of GRACE scores on prediction of 30-day cardiovascular adverse events in acute chest pain patients. A prospective, observational analysis was conducted in the patients with acute chest pain in Emergency Department (ED) from January 1, 2016 through January 1, 2017. Data including characteristics and GRACE scores were collected. All causes leading to MACE were followed up at 30th day after the onset of acute chest pain. Among a total of 600 patients presenting with acute chest pain enrolled in this study, 302 were male $(50.3 \%)$ and 298 were female $(49.7 \%)$. The range of age was 20-80 years old. During follow-up period, 102 patients had MACE, 498 patients had no MACE. When compared with non-MACE group, factors including number of Smoker, Hypercholesterolemia, Diabetes, Hypercholesterolemia and patients admitted in CCU as well as GRACE scores, were significantly higher in MACE group $(P<0.05)$. The predictive ROC curve area of GRACE scores in 30-day MACE was 0.739 ( 0.687 to 0.791$)$. The probability of 30-day cardiovascular adverse events in various GRACE score risk stratification was $2.0 \%$ (low-risk), $5.33 \%$ (medium-risk), and 9.67\% (high-risk), respectively. The GRACE score was a useful predictor to the occurrence of 30-day cardiovascular adverse events in acute chest pain patients. Patients with low GRACE score risk stratification have a low risk of 30-day MACE, which may be able to convey risk quickly and efficiently.
\end{abstract}

Keywords: GRACE Scores, Acute Chest Pain, Risk Stratification, Cardiovascular Adverse Events

\section{Introduction}

Acute chest pain is one of the most common symptoms in the emergency department (ED). More than 8 million people in the United States visit chest pain every year, and about $10 \%$ to $15 \%$ of myocardial infarctions [1-2]. Due to the high-risk factors of acute chest pain, especially ACS, the clinical manifestations are not the same. Myocardial infarction occurred in $2 \%$ to $4 \%$ of patients who returned home with only ECG and myocardial markers [3]. For emergency physicians, it is important to have a risk stratification of these patients and to accurately identify high-risk patients and triage patients early. Therefore, clinically, acute chest pain patients urgently need a quick and reliable risk assessment method to conduct risk stratification to improve the overall diagnosis and treatment level of the ED [4]. Currently GRACE score is one of the effective risk stratification methods for ACS. The GRACE score was initially created by Granger et al which the main variables include eight factors such as age, blood pressure, heart rate, which are derived from the largest multinational multicenter prospective registration study [5]. As of March 2011, there were 247 hospitals in more than 30 countries and 102341 patients of ACS were enrolled in the study. GRACE score for all ACS patients hospitalized and 6 months of major cardiovascular adverse events (MACE) have good predictive value. The recent ACC/AHA treatment guidelines also recommend the GRACE score as one of the primary criteria for the risk assessment at admission and 
discharge of ACS patients and outside hospital [6]. However, there is no data study on the value of GRACE score in assessing the prognosis of patients with acute chest pain in ED. Therefore, this study intends to conduct GRACE scores on 209 patients with chest pain who were admitted to the ED of the First Affiliated Hospital of Sun Yat-sen University from January to April 2016 to explore the predictive value of 30-day MACE in patients with acute chest pain to improve the corresponding judgment ability and the level of diagnosis and treatment of clinicians.

\section{Patients and Methods}

\subsection{Research Subjects}

Continuous collection of ED of the First Affiliated Hospital of Sun Yat-sen University 600 patients who were mainly complained of "chest pain and chest tightness" from January 1, 2016 to January 1, 2017 (mainly diagnosed as ACS, aortic dissection, pulmonary embolism, pneumothorax, gastroesophageal reflux, valvular disease, intercostal neuritis, etc.), including 325 males and 275 females, aged (64.6 \pm 16.85$)$ years old. According to whether MACE is divided into 102 cases in the MACE group and 498 cases in the non-MACE group. This study has been approved by the Ethics Committee of the First Affiliated Hospital of Sun Yat-sen University.

\subsubsection{Inclusion Criteria}

i. age $\geq 18$ and $<80$ years old; ii. visiting the ED; iii. meet any of the following two: 1). The main complaint is acute chest pain, including crushing pain, acupuncture pain, dull pain, burning pain, stuffy pain, distending pain and other pains of all nature, 2): the first doctor suspected ACS (according to the 2014 AHA/ACC guidelines recommended ACS diagnostic criteria); iv. patients within 24 hours before the visit; e) signed informed consent.

\subsubsection{Exclusion Criteria}

a) Chest pain caused by trauma; b) systemic pain caused by cancer and rheumatic diseases involves the chest; c) Patients who were visited again within 30 days of the initial visit.

\subsection{General Information}

Medical history data collection: Communicate with all selected patients or their families and sign an informed consent form, ask in detail about past medical history and completed physical examination (especially heart and lung), and recorded the general condition of the patient (age, gender, hypertension, diabetes, smoking, drinking, past medical history, etc) and basic vital signs (blood pressure, pulse, oxygen saturation, respiration) during emergency triage. Completed blood routine, troponin (TnT, cTnI), pro-BNP, electrolytes, liver and kidney function, D-dimer and other tests. All the enrolled patients were enrolled for the occurrence of MACE (All due to death, myocardial infarction, emergency revascularization, cardiogenic shock, cardiac arrest, ventricular fibrillation, stroke) during the 1-month follow-up.

\subsection{GRACE Scoring System}

According to the age, heart rate, systolic blood pressure, creatinine and Killip classification of all patients with acute chest pain, GRACE scores were scored. The scores $<108$ were classified as low-risk, $109-140$ as high-risk, and $\geq 140$ as high-risk. The specific scores are shown in Table 1.

Table 1. The system of GRACE risk score.

\begin{tabular}{|c|c|c|c|c|c|c|c|c|c|c|c|}
\hline $\begin{array}{l}\text { Age } \\
\text { (year) }\end{array}$ & Score & $\begin{array}{l}\text { Heart rate } \\
\text { (bpm) }\end{array}$ & Score & $\begin{array}{l}\text { SPB } \\
(\mathrm{mmHg})\end{array}$ & $\begin{array}{l}\text { Scor } \\
\text { e }\end{array}$ & $\mathbf{C r}$ & Score & $\begin{array}{l}\text { Killip } \\
\text { class }\end{array}$ & Score & Others & Score \\
\hline$<30$ & 0 & $<50$ & 0 & $<80$ & 58 & 35 & 1 & class I & 0 & Elevated cardiac makers & 14 \\
\hline $30 \sim 39$ & 8 & $50 \sim 69$ & 3 & $80 \sim 99$ & 53 & $36 \sim 70$ & 4 & class II & 20 & ST-segment deviation & 28 \\
\hline 40 49 & 25 & $70 \sim 89$ & 9 & $100 \sim 119$ & 43 & $71 \sim 105$ & 7 & class III & 39 & Cardiac arrest at admission & 39 \\
\hline $50 \sim 59$ & 41 & $90 \sim 109$ & 15 & $120 \sim 139$ & 34 & $106 \sim 140$ & 10 & class IV & 59 & & \\
\hline $60 \sim 69$ & 58 & $110 \sim 149$ & 24 & $140 \sim 159$ & 24 & $141 \sim 175$ & 13 & & & & \\
\hline $80 \sim 89$ & 91 & $>200$ & 46 & $\geq 200$ & 0 & $\geq 352$ & 28 & & & & \\
\hline$\geq 90$ & 100 & & & & & & & & & & \\
\hline
\end{tabular}

\subsection{Data Statistics Method}

All data was recorded through double entry of EpiData 3.1 software. Data correction was performed on the original questionnaire if incorrect data input was found. The database was then loaded into SPSS 22.0 software for data filtering, collation, and analysis. The measurement data were expressed as mean $\pm \mathrm{SD}$. The comparison between the two groups was performed by independent sample $t$ test. The count data were expressed by frequency or rate, and the differences between groups were analyzed by chi-square test or Fisher test. All of the above statistical tests are two-sided tests. $\mathrm{P}<0.05$ was considered statistically significant. The ROC curve for the 30-day prognosis of patients with acute chest pain was assessed by GRACE scores.

\section{Results}

\subsection{Research Target Population Flow Chart}

As shown in figure 1, 600 patients with acute chest pain were included in this study, including 498 in the control group and 102 in the MACE group. Among them, 98 patients with AMI, 79 patients accepted PCI, 18 patients died after hospitalization or go home, and 1 patient get CABG. 


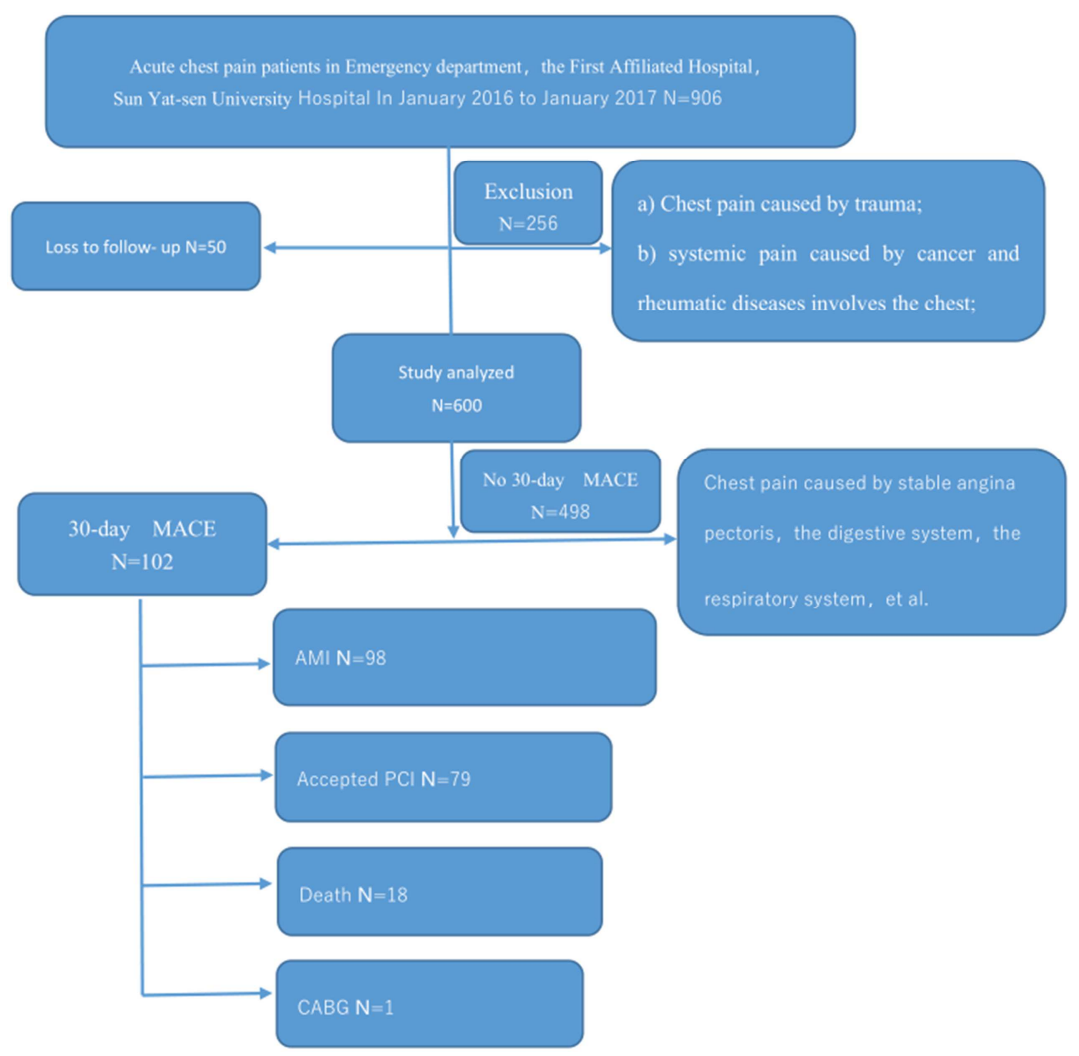

Figure 1. Patient flow chart and breakdown of primary outcome of 30-day major adverse cardiac events (MACE). MACE: cardiovascular adverse events AMI: acute myocardial infarction; PCI: percutaneous coronary stent implantation CABG: coronary artery bypass grafting.

\subsection{Analysis and Comparison of General Data of Selected Patients}

The comparison of basic clinical data between MACE group and non-mace group is shown in table 2: The age range of patients enrolled in the study ranged from 20 to 80 years old, with an average of $(66.6 \pm 16.85)$ years old, 302 males $(50.33 \%)$ and 298 females (49.67\%). Clinical baseline information includes age, sex, body mass index (BMI), past medical history (smoking, coronary heart disease, myocardial infarction, diabetes, hypertension), admission (blood pressure, pulse, $\mathrm{SaO}$ ), hospitalization. The history of smoker, hypercholesterolemia, diabetes, hypercholesterolemia and the number of cases $\mathrm{CCU}$ in the MACE group, GRACE scores were higher than those in the non-MACE group, and the difference was statistically significant $(\mathrm{P}<0.05)$; However, the clinical data of the MACE group and the non-MACE group showed no significant difference $(\mathrm{P}>0.05)$ in male patients, past medical history (coronary heart disease, myocardial infarction), blood pressure at admission, etc.

Table 2. Comparison of Clinical Baseline Data between MACE and non-MACE.

\begin{tabular}{lll}
\hline & MACE gourp (n=102) & non-MACE group (n=498) \\
\hline Age, year, mean (SD) & $76.3(8.4)$ & $64.6(17.0)$ \\
Male gender, n (\%) & $54(52.9)$ & $248(49.8)$ \\
BMI, kg/m ${ }^{2}$ & $24.35(3.4)$ & $22.72(3.2)$ \\
Previous history & & \\
Smoker, $\mathrm{n}(\%)$ & $23(22.5)$ & $68(13.7) \#$ \\
Hypercholesterolemia, n (\%) & $43(42.2)$ & $174(34.9) \#$ \\
History of CAD, n (\%) & $23(22.5)$ & $53(10.6)$ \\
History of AMI, n (\%) & $5(4.9)$ & $22(4.4)$ \\
Diabetes, n (\%) & $45(44.1)$ & $62(12.5) \#$ \\
Hypercholesterolemia, n (\%) & $35(34.3)$ & $77(15.5) \#$ \\
vital signs at admission & & \\
SBP, mmHg, mean (SD) & $131.4(39.3)$ & $151.8(27.8)$ \\
DBP, mmHg, mean (SD) & $73.8(12.5)$ & $82.9(16.4)$ \\
Heart rate, mean (SD) & $84.5(22.5)$ & $84.7(18.7)$ \\
SPO2 (\%) mean (SD) & $94.9(4.9)$ & $97.4(2.3)$ \\
CCU, $\mathrm{n}(\%)$ & $67(65.7)$ & $48(9.6) \#$ \\
GRACE score, mean (SD) & $140.3(38.0)$ & $109.2(33.5) \#$ \\
\hline
\end{tabular}

MACE: cardiovascular adverse events, BMI: body mass index, CAD: coronary heart disease, SBP: systolic blood pressure, DBP: diastolic blood pressure, CCU: cardiac care unit, AMI: acute myocardial infarction; GRACE: global registry of acute coronary events. ${ }^{~} \mathrm{P}<0.05$ vs. control. 


\subsection{GRACE Score Prediction Value}

The ROC curve of GRACE risk score predicting the occurrence of MACE on 30 days of acute chest pain showed that when GRACE score $=118$, the maximum area under the curve was 0.739 (95\%ci: 0.687-0.791).

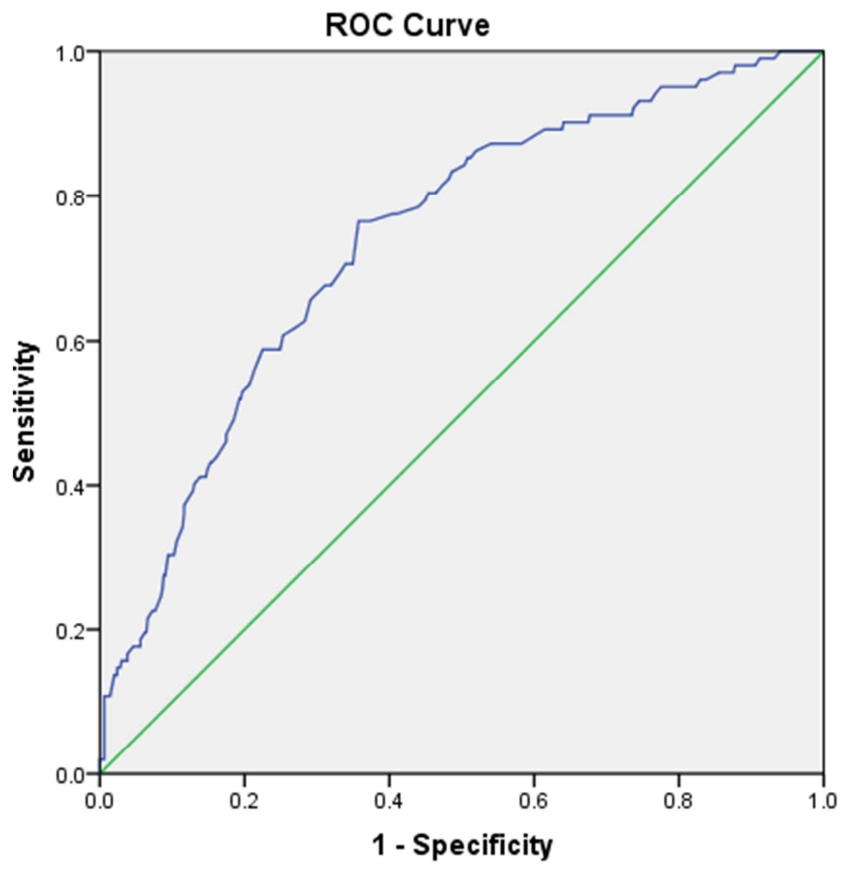

Diagonal segments are produced by ties.

Figure 2. Receiver operating characteristic (ROC) curve for the GRACE score in predicting 30-day major adverse cardiac events (MACE).

\subsection{The rate of MACE in Different GRACE Scores Risk Stratification}

According to the GRACE scoring criteria, they were divided into low-risk ( $<108$ points), medium-risk (109-140 points) and high-risk ( $>140$ points). Just as showed in Table 3 , 273 patients in low-risk, 193 patients in medium-risk and 134 patients in high-risk. The probability of 30-day cardiovascular adverse events in various GRACE score risk stratification was $2.0 \%$ (low-risk), 5.33\%(medium-risk), and 9.67\% (high-risk), respectively.

Table 3. The rate of MACE in different GRACE scores risk stratification.

\begin{tabular}{lll}
\hline Classification & Tatal $\mathbf{n}$ & MACE $\mathbf{n}(\%)$ \\
\hline High class & 134 & $58(9.67)$ \\
Medium class & 193 & $32(5.33)$ \\
Low class & 273 & $12(2.0)$ \\
\hline
\end{tabular}

\section{Discussion}

In recent years, the number of patients with acute chest pain has increased significantly, and ED patients complained of chest pain accounted for $20 \% \sim 40 \%$ [7-8]. There are many kinds of diseases that cause acute chest pain, and symptoms are different, it is easy to miss diagnosis and misdiagnosis.
Acute chest pain in critical diseases, such as acute coronary syndrome, pulmonary embolism and aortic dissection, is highly fatal and disabling if it cannot be recognized and treated in time [9-11]. Some patients with mild symptoms often have over-examination, which brings heavy financial and mental burden to patients and their families. The GRACE score was validated from a large-scale multicenter registry study for all ACS patients [12-13]. In recent years, many studies have found that the GRACE score can also be used for the assessment of risk factors in patients with acute chest pain, and has a certain predictive value for hospitalization and discharge prognosis [14-15]. Therefore, this study sought to provide a new basis for clinically relevant applications by exploring the predictive effect of GRACE score on 30-day MACE in emergency patients with chest pain.

The study found that compared with the non-MACE group, the GRACE score was significantly higher in the MACE group, and the age, BMI, hospitalization, and CCU hospitalization ratios in the MACE group were higher than those in the control group; By plotting the ROC curve of the prediction of MACE by GRACE score, it was found that the GRACE score had a better prediction effect on MACE for acute chest pain at 30 days. as the GRACE score was higher, the probability of a 30-day MACE was greater.

Studies have reported that foreign assessments of acute chest pain are mostly directed to a single disease, such as an aortic dissection (AD) risk score for early assessment of AD. Wells score for early prediction of pulmonary embolism, and traditional GRACE score for the risk stratification and prognosis assessment of ACS patients, all of these have good value. These scores can quickly and effectively judge the patient's condition and triage, and have a certain guiding effect on the chest pain unit. However, the high-risk diseases that cause acute chest pain in the ED include ACS, AD, pulmonary embolism, and tension pneumothorax. The above simple scores are not fully applicable to all patients with acute chest pain. Through this study, we first discovered that the GRACE score can effectively predict 30-day MACE in patients with acute chest pain. This finding just makes up for the shortcomings of traditional scoring and applies to chest pain caused by various causes. With the increases of GRACE score, the probability of MACE risk also increases. Therefore, it is recommended to be highly vigilant and actively deal with it clinically, and to establish a smooth green channel when necessary to provide better services for patients, save their lives to the greatest extent and reduce their psychological and economic burden.

\section{Conclusions}

In summary, the GRACE score in our study has a clinical guiding effect on the predictive evaluation of 30 days MACE in patients with acute chest pain. Patients with low GRACE score risk stratification have a low risk of 30-day MACE, which may be able to convey risk quickly and efficiently. 


\section{Limitations}

However, there are still some limitations: 1) In this study, due to the small sample size, limited number of indicators, and many clinical confounding factors affecting MACE. So, the recent prognostic evaluation cannot completely eliminate the bias of results caused by other factors, and the sample size needs to be further expanded; 2) The study participants were mainly from southern regions, with a wide geographical areas and differences in ethnicity, a national multicenter study is needed to further confirm its clinical value in patients with acute chest pain.

\section{List of Abbreviations}

MACE: cardiovascular adverse events AMI: acute myocardial infarction; PCI: percutaneous coronary stent implantation $\mathrm{CABG}$ : coronary artery bypass grafting, BMI: body mass index, CAD: coronary heart disease, SBP: systolic blood pressure, DBP: diastolic blood pressure, CCU: cardiac care unit, AMI: acute myocardial infarction; GRACE: global registry of acute coronary events, ACS: acute coronary syndrome.

\section{Acknowledgements and Funding}

The study was financially supported by the project of science and technology program of Guangzhou City (201803010008 and 201804010007), and the international scientific and technological cooperation project of Guangzhou Economic and Technological Development Zone (2017GH13).

\section{References}

[1] Poldervaart JM, Langedijk M, Backus BE et al. Comparison of the GRACE, HEART and TIMI Score to Predict Major Adverse Cardiac Events in Chest Pain Patients at the Emergency Department. Int J Cardiol. 2017; 227: 656-661.

[2] Rahko PS. Rapid Evaluation of Chest Pain in the Emergency Department. Jama Intern Med. 2014; 174 (1): 59-60.

[3] Holly J, Fuller M, Hamilton D et al. Prospective Evaluation of the Use of the Thrombolysis in Myocardial Infarction Score as a Risk Stratification Tool for Chest Pain Patients Admitted to an ED Observation Unit. Am J Emerg Med. 2013; 31 (1): 185-189.

[4] Sakamoto JT, Liu N, Koh ZX et al. Comparing HEART, TIMI, and GRACE Scores for Prediction of 30-Day Major Adverse Cardiac Events in High Acuity Chest Pain Patients in the Emergency Department. Int J Cardiol. 2016; 221: 759-764.

[5] Granger C B, Goldberg R J, Dabbous O, et al. Predictors of hospital mortality in the global registry of acute coronary events. [J]. Arch Intern Med. 2003; 163 (19): 2345-2353.

[6] Six A J, Backus B E, Kelder J C. Chest pain in the emergency room: value of the HEART score. [J]. Neth Heart J. 2008; 16 (6): 191-196.

[7] Cotterill PG, Deb P, Shrank WH, Pines JM. Variation in Chest Pain Emergency Department Admission Rates and Acute Myocardial Infarction and Death within 30 Days in the Medicare Population. Acad Emerg Med. 2015; 22 (8): 955-964.

[8] Ornato JP. Chest Pain Emergency Centers: Improving Acute Myocardial Infarction Care. Clin Cardiol. 1999; 8 Suppl: V3-9.

[9] S L, L S, L S, et al. Different Causes of Death in Patients with Myocardial Infarction Type 1, Type 2, and Myocardial Injury. Am J Med. 2018; 131 (5): 548-554.

[10] Roberts Lara N., Whyte Martin B., Arya Roopen. Pulmonary embolism mortality trends in the European region-too good to be true? Lancet Respir Med. 2020; 8 (1), e2.

[11] E M. Half of patients with acute aortic dissection in England die before reaching a specialist centre. BMJ (Clinical research ed.). 2020; 368: m304.

[12] Zhou B, Zu L, Mi L, et al. An analysis of patients receiving emergency CAG without PCI and the value of GRACE score in predicting PCI possibilities in NSTE-ACS patients. [J]. $J$ Geriatr Cardiol., 2015, 12 (3): 246-250.

[13] Ang DS, Wei L, Kao MP, et al. A comparison between B-type natriuretic peptide, global registry of acute coronary events (GRACE) score and their combination in ACS risk stratification. Heart, 2009; 95 (22): 1836-42.

[14] Reaney PDW, Elliott HI, Noman A, Cooper JG. Risk Stratifying Chest Pain Patients in the Emergency Department Using HEART, GRACE and TIMI Scores, with a Single Contemporary Troponin Result, to Predict Major Adverse Cardiac Events. Emerg Med J. 2018; 7: 420-7.

[15] Backus BE, Six AJ, Kelder JC et al. A Prospective Validation of the HEART Score for Chest Pain Patients at the Emergency Department. Int J Cardiol. 2013; 3: 2153-8. 